\title{
Pyridoxamine and Caloric Restriction Improve Metabolic and Microcirculatory Abnormalities in Rats with Non-Alcoholic Fatty Liver Disease
}

\author{
Evelyn Nunes Goulart da Silva Pereira Raquel Rangel Silvares \\ Karine Lino Rodrigues Edgar Eduardo llaquita Flores Anissa Daliry \\ Laboratory of Cardiovascular Investigation, Oswaldo Cruz Institute, FIOCRUZ, Rio de Janeiro, Brazil
}

\section{Keywords}

Pyridoxamine $\cdot$ Caloric restriction $\cdot$ Microvascular blood

flow · Metabolic diseases · Liver diseases

\begin{abstract}
Introduction: This study aims to examine the effect of a diet intervention and pyridoxamine (PM) supplementation on hepatic microcirculatory and metabolic dysfunction in nonalcoholic fatty liver disease (NAFLD). Methods: NAFLD in Wistar rats was induced with a high-fat diet for 20 weeks (NAFLD 20 weeks), and control animals were fed with a standard diet. The NAFLD diet intervention group received the control diet between weeks 12 and 20 (NAFLD 12 weeks), while the NAFLD 12 weeks + PM group also received PM. Fasting blood glucose (FBG) levels, body weight (BW), visceral adipose tissue (VAT), and hepatic microvascular blood flow (HMBF) were evaluated at the end of the protocol. Results: The NAFLD group exhibited a significant increase in BW and VAT, which was prevented by the diet intervention, irrespective of PM treatment. The FBG was elevated in the NAFLD group, and caloric restriction improved this parameter, although additional improvement was achieved by PM. The NAFLD group displayed a $31 \%$ decrease in $\mathrm{HMBF}$, which was par-
\end{abstract}

tially prevented by caloric restriction and completely prevented when PM was added. HMBF was negatively correlated to BW, FBG, and VAT content. Conclusion: PM supplementation in association with lifestyle modifications could be an effective intervention for metabolic and hepatic vascular complications.

(c) 2021 S. Karger AG, Basel

\section{Introduction}

A sedentary lifestyle, coupled with increased consumption of fat-laden, high-calorie diets, contributes to the growing prevalence of obesity worldwide [1]. Globally, obesity has become one of the main clinical and public health problems. According to the World Health Organization, $39 \%$ of adults aged 18 years or older are overweight, while $13 \%$ are obese $[2,3]$. Obesity is a multifactorial chronic disorder associated with cardiac and metabolic complications influenced by genetic factors, hormones, environment, and diet [4].

Along with increasing obesity rates, the incidence and prevalence of nonalcoholic fatty liver disease (NAFLD) continues to rise, affecting $25-30 \%$ of the adult popula- karger@karger.com

www.karger.com/jvr

Karger ${ }^{\prime}=$

(C) 2021 S. Karger AG, Basel 
tion [5]. NAFLD encompasses a wide spectrum of conditions ranging from simple steatosis, characterized by triglyceride accumulation in the liver, to nonalcoholic steatohepatitis, the inflammatory form of the disease that can lead to progressive liver damage, which may result in cirrhosis and hepatocellular carcinoma [6]. Up to $80 \%$ of patients with NAFLD are obese [7, 8]. Fatty liver severity in patients with morbid obesity is also correlated to the degree of impaired glycemic status [9]. The amount and distribution of visceral adipose tissue (VAT) plays a greater role in insulin resistance than the BMI [10] and is positively related to the presence of NAFLD [10]. Excess fatty acids derived from VAT tissue via lipolysis, as well as from dietary sources and de novo lipogenesis, are released to the portal venous system [11] and cause chronic low-grade inflammation which then participates in liver injury progression in NAFLD $[12,13]$. Although obesity is the major risk factor for NAFLD development and progression $[14,15]$, the exact molecular and pathophysiological insults that contribute to NAFLD progression are not yet fully understood.

Obesity has been implicated in the pathogenesis of both macro- and microvascular abnormalities [16-18]. Human and animal studies demonstrate that obesity is associated with systemic and hepatic endothelial dysfunction [19-21] and increased cardiovascular disease $[22,23]$. Regional and systemic hemodynamic pathological changes contribute to an altered hepatic microcirculation flow, subsequently affecting hepatocellular function [24]. Fatty accumulation in the hepatocyte cytoplasm is associated with increased cell volume, which may result in partial or complete obstruction of the hepatic sinusoid space which, in turn, decreases the hepatic blood flow [25, 26]. Decreases in the microcirculatory hepatic blood flow may enhance hepatic injury, impairing exchanges between the blood and the vasculature and depriving hepatocytes of oxygen and nutrients [27]. Thus, microcirculatory alterations are an important hit in NAFLD progression, emerging as a promising pharmacological therapy target for NAFLD management.

The current scenario for the control and management of NAFLD is complex, since there are no approved pharmacological treatments, and the recommendations relies on lifestyle changes, including physical activity and caloric restriction, and control of risk factors, which is not easy to be reached since the adherence and maintenance of these strategies is not a trivial task, neither on the part of the patients nor on the part of public policies [28-34]. Caloric restriction must be associated with considerable weight loss (i.e., 7-10\%) in order to achieve important ef- fects on steatosis and other liver markers, which makes it even more difficult to be achieved by individuals $[35,36]$. Therefore, the search for new intervention strategies that help reduce the impact of NAFLD on global public health and slow the progress of the disease to more severe cases is of great importance.

Nonpharmacological interventions have emerged as promising novel therapeutic agents in promoting antiinflammatory, antioxidative, and antifibrotic effects, with less adverse effects and, consequently, high treatment adherence [37]. Pyridoxamine (PM), a vitamin B6 analog, has been reported to significantly improve blood glucose levels [38] and attenuate high-fat diet (HFD)-induced weight gain and VAT hypertrophy $[39,40]$. Our previous data indicate that PM treatment is able to revert HFDinduced hepatic vascular dysfunction [20] through antiglycating, antioxidative, and anti-inflammatory mechanisms [21]. These findings highlight the potential of PM as an intervention strategy in obesity and NAFLD. Therefore, the present study examined the effect of a diet intervention, that is, reduced caloric intake, on hepatic microcirculatory dysfunction and metabolic complications in HFD-induced obese mice, a diet-induced obese animal model with NAFLD features [20]. In addition, PM treatment was combined with a diet intervention in order to verify if both treatments result in additional improvements when compared to their isolated effects.

\section{Materials and Methods}

\section{Experimental Protocol and Animals}

Four-week-old male Wistar rats were housed in polypropylene cages and maintained in a controlled room ( $12 \mathrm{~h}$ light/dark cycle and constant temperature of $22 \pm 1^{\circ} \mathrm{C}$ ). The animals were randomly divided into 4 experimental groups: the non-NAFLD control (CTL) group, which remained on ad libitum regular chow (Nuvilab - CR1; Nuvital Nutrients Ltd) throughout the whole experiment (CTL; $n=10$ ); the HFD-induced NAFLD group, which had free access to a diet with saturated fat (lard) as the main fat source $(30 \% \mathrm{~g} / \mathrm{fat})$ during 20 weeks (NAFLD 20 weeks; $n=10$ ); the NAFLD diet intervention group, which received the HFD until week 12 and the normocaloric control diet between weeks 12 and 20 (NAFLD 12 weeks; $n=10$ ); and the NAFLD diet intervention group, which, in addition to, being submitted to the diet intervention, received PM treatment (PrimAGE; Life Link, Tampa, FL, USA) $(60 \mathrm{mg} / \mathrm{kg} /$ day, v.o. by gavage) between weeks 12 and 20 (NAFLD 12 weeks + PM). Food intake was measured every 3 days. At the end of the protocol, the animals were anesthetized and hepatic microvascular blood flow (HMBF) assessed by laser speckle contrast imaging (LSCI) (Fig. 1). Body weight (BW) was measured, and abdominal and epididymal adipose tissue deposits were dissected and weighed. All experiments were conducted in accordance with 
Fig. 1. Schematic representation of the experimental design. Forty 4-week-old male Wistar rats were randomly assigned to 4 groups, namely, the non-NAFLD control group, which remained on ad libitum regular chow throughout the whole experiment (CTL); the HFD-induced NAFLD group, which had free access to a diet with saturated fat (lard) as the main fat source (30\% $\mathrm{g} /$ fat) during 20 weeks (NAFLD 20 weeks); the NAFLD diet intervention group, which received the HFD until week 12 and the control normocaloric control diet between weeks 12 and 20 (NAFLD 12 weeks); and the NAFLD diet intervention group, which, besides being submitted to the diet intervention, received PM treatment $(60 \mathrm{mg} / \mathrm{kg} /$ day, v.o. by gavage) between weeks 12 and 20 (NAFLD 12 weeks + PM). At the end of the protocol, the animals were anesthetized, hepatic microvascular blood flow was assessed by LSCI, FBG and BW were measured, and abdominal and epididymal adipose tissue deposits were dissected and weighed. LSCI, laser speckle contrast imaging; PM, pyridoxamine; CTL, control; FBG, fasting blood glucose; BW, body weight; HFD, high-fat diet; NAFLD, nonalcoholic fatty liver disease.

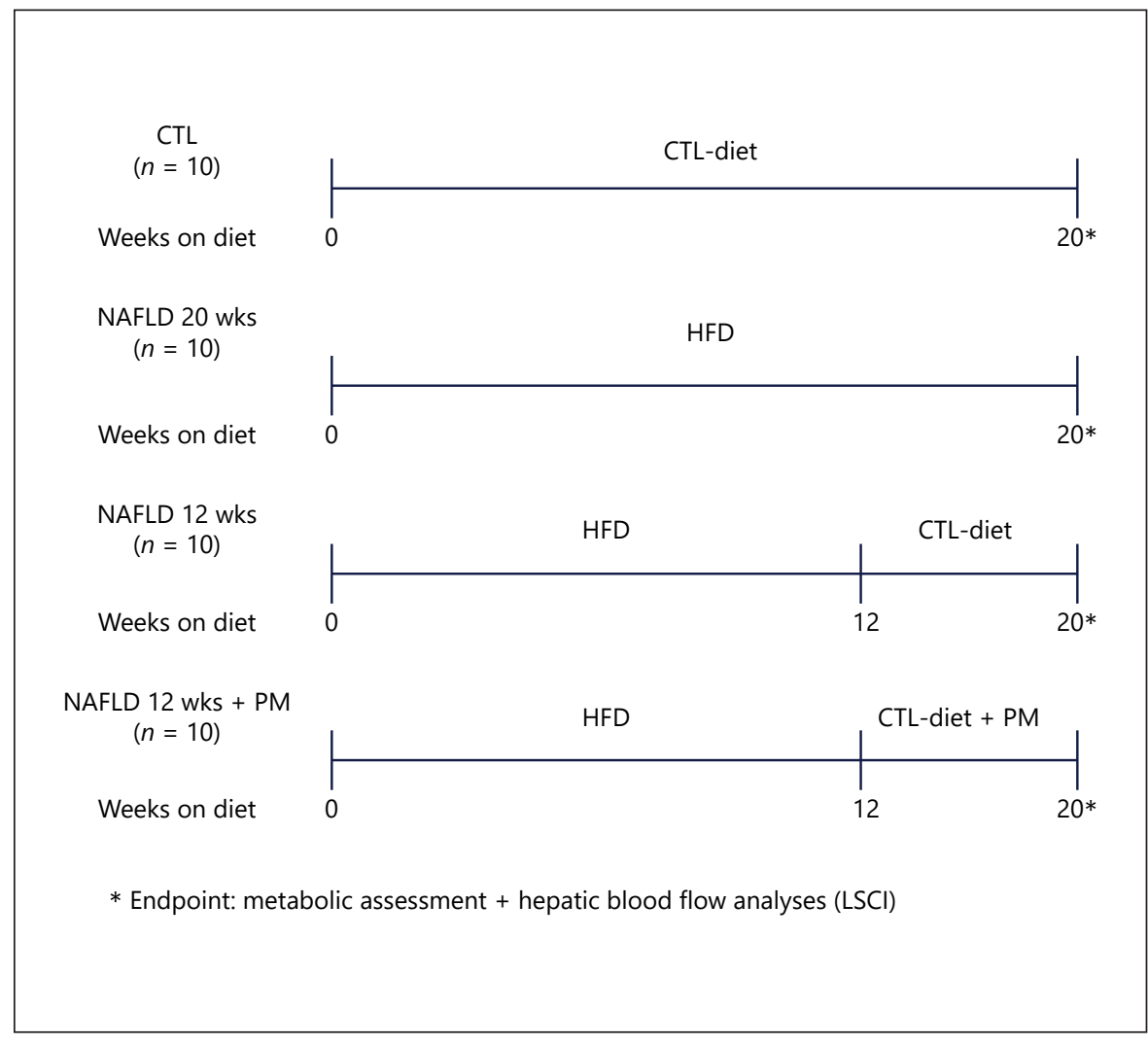

internationally accepted principles for the care and use of laboratory animals and were approved by the Oswaldo Cruz Foundation Animal Welfare Committee (License L-019/2016).

\section{Fasting Blood Glucose Measurement}

Fasting blood glucose (FBG) levels were measured using a glucometer (Accu-Chek; Roche, Sao Paulo, SP, Brazil) in animals that fasted overnight. Using surgical scissors, a cut was made $<1 \mathrm{~mm}$ from the tip of the tail, obtaining a drop of blood collected on the tape previously inserted in the glucometer. FBG was expressed as $\mathrm{mmol} / \mathrm{L}$.

\section{Adipose Tissue}

Abdominal and epididymal adipose tissue deposits were dissected after the euthanasia. Epididymal fat was considered as the fat present in the lower part of the abdomen connected to the epididymis. Abdominal fat was considered as the whole fat pads adhered to the intestines. After dissection, the adipose tissue deposits were weighed using an analytical balance (GR-200; A\&D Company Limited, Tokyo, Japan).

\section{Food and Energy Intake}

Free access to food and water was provided during the experimental protocol. Food intake was measured every 3 days using a balance (UDC 50000/20; Urano, Brazil), determined as the difference between the offered and nonconsumed feed. Food consumption was expressed as the daily food intake, in grams (g), and daily energy intake, in kilocalorie (kcal), with the energy intake determined as the product of food consumption by the dietary energy content.

\section{Laser Speckle Contrast Imaging}

The laser speckle contrast imaging apparatus (Pericam PSI system, Perimed, Sweden), a real-time noninvasive and noncontact modality for the monitoring of microvascular blood flow and perfusion, was used to access the perfusion of the liver basal microvascular blood flow. The technique provides a microcirculatory perfusion index proportional to the moving scattering particles in the tissue (e.g., RBCs) $[20,41,42]$. For this analysis, animals were fasted overnight, anesthetized intraperitoneally with ketamine $(100 \mathrm{mg} / \mathrm{kg}$, i.p.) and xylazine (10 mg/kg, i.p.), and a laparotomy was performed. The animals were maintained in a room with a constant temperature at $25^{\circ} \mathrm{C}$ and a stable surface. The left lobe of the liver was placed on a glass disk and placed under a laser light system with image contrast for acquisition of the continuous measurement of tissue blood perfusion data, accomplished with a laser wavelength of $785 \mathrm{~nm}$. The distance between the camera and the liver was set to $10.0 \mathrm{~cm}$. Relative $\mathrm{HMBF}$ of the animals was expressed as arbitrary perfusion units (APUs).

\section{Statistical Analyses}

The results were expressed as means \pm SEM for each group. Normal distribution was assessed using the Shapiro-Wilk test, and comparisons between groups were performed using a one-way ANOVA, followed by Bonferroni post hoc tests for multiple comparisons to follow up significances and interactions. The data were analyzed using GraphPad Prism, v. 8.0.1, software (GraphPad Software Inc., LA Jolla, CA, USA). The Pearson correlation coefficient was used to assess correlations between metabolic and microcirculatory parameters ( $R$ Studio-version 4.0.2). Values set at $p<0.05$ were considered significant. 
Table 1. Description of the diets used in the CTL and HFD groups

\begin{tabular}{lcc}
\hline g/100 g of diet (\%kcal) & CTL & HFD \\
\hline Protein & $21.4 \%$ & $10.2 \%$ \\
Commercial chow & 23 & 13.2 \\
Condensed milk & - & 1.4 \\
\hline Carbohydrates & $66 \%$ & $40.7 \%$ \\
Commercial chow & 71 & 16 \\
Corn starch & - & 30 \\
Condensed milk & - & 10 \\
\hline Fat & $12.6 \%$ & $49.1 \%$ \\
Commercial chow & 6 & 2.5 \\
Condensed milk & - & 1.6 \\
Animal fat (lard) & - & 25 \\
\hline \%Kcal/kg & 4,300 & 5,500 \\
\hline
\end{tabular}

CTL, control; HFD, high-fat diet.

\section{Results}

\section{Obesity Phenotype}

The metabolic and hemodynamic characteristics of the NAFLD animal model have been previously described in detail [20]. The diet composition used in the present study is depicted in Table 1. HFD administration did not induce alterations in daily calorie intake when compared to normal chow (Table 2), as, although the HFD comprises a higher caloric content than the CTL diet, animals fed on HFD exhibited reduced daily food intake.

Free access to a saturated HFD for 20 weeks led to an obesity-like phenotype, as observed in the NAFLD 20 week group (Fig. 2). The HFD induced a 9\% increase in BW compared to the CTL group (CTL: $481.9 \pm 10.36 \mathrm{~g}$ vs. NAFLD 20 weeks: $525.2 \pm 12.91 \mathrm{~g}, p=0.017$ ), while the diet intervention alone or associated with PM treatment prevented BW increases (NAFLD 12 weeks: $455.2 \pm 11.17$ $\mathrm{g}$ and NAFLD 12 weeks + PM: $442.9 \pm 7.93 \mathrm{~g}$ vs. NAFLD 20 weeks: $525.2 \pm 12.91 \mathrm{~g}, p<0.001$ ) (Fig. $2 \mathrm{a}$ ). Abdominal fat content was $45 \%$ higher in the NAFLD 20 week animal group than that in the CTL group (CTL: $5.34 \pm 0.46 \mathrm{~g}$ vs. NAFLD 20 weeks: $7.75 \pm 0.63 \mathrm{~g}, p=0.002$ ) (Fig. 2b). The diet interventions with or without PM treatment were similarly effective in reducing abdominal fat content (NAFLD 12 weeks: $4.29 \pm 0.38 \mathrm{~g}$ and NAFLD 12 weeks + PM: $3.77 \pm 0.38$ g vs. NAFLD 20 weeks: $7.75 \pm 0.63 \mathrm{~g}, p<$ 0.001 ) (Fig. 2b). Epididymal fat content exhibited a $74 \%$ increase in the NAFLD 20 week group when compared to the CTL group (CTL: $10.37 \pm 0.90$ g vs. NAFLD 20 weeks: $18.06 \pm 1.38 \mathrm{~g}, p=0.001$ ), while caloric restriction, alone
Table 2. Description of the daily food intake in the CTL and HFD groups

\begin{tabular}{llll}
\hline & CTL & HFD & $p$ value \\
\hline Daily food intake, g & $18.0 \pm 1.7$ & $15.0 \pm 0.5$ & 0.045 \\
Daily energy intake, kcal & $79.0 \pm 7.3$ & $84.0 \pm 3.2$ & 0.443 \\
\hline
\end{tabular}

Values are expressed as means \pm SEM. CTL, control; HFD, high-fat diet.

Table 3. Correlation between metabolic and microcirculatory parameters evaluated by Pearson's correlation analysis

Pearson correlation $p$ value coefficient $(r)$

\begin{tabular}{lcr} 
Liver microvascular blood flow & & \\
BW & -0.39 & 0.012 \\
FBG & -0.35 & 0.024 \\
Epididymal fat content & -0.48 & 0.001 \\
Abdominal fat content & -0.43 & 0.006 \\
\hline$B W$ & & \\
FBG & 0.31 & 0.056 \\
Epididymal fat content & 0.83 & $<0.001$ \\
Abdominal fat content & 0.78 & $<0.001$ \\
\hline FBG & & \\
Epididymal fat content & 0.33 & 0.041 \\
Abdominal fat content & 0.18 & \\
\hline Epididymal fat content & & \\
Abdominal fat content & 0.88 & \\
\hline
\end{tabular}

FBG, fasting blood glucose; BW, body weight.

or in association with PM treatment, reduced epididymal fat content in the same degree (NAFLD 12 weeks: $9.46 \pm$ $0.99 \mathrm{~g}$ and NAFLD 12 weeks + PM: $9.58 \pm 0.88 \mathrm{~g}$ vs. NAFLD 20 weeks: $18.06 \pm 1.38 \mathrm{~g}, p=0.001$ ) (Fig. 2c).

FBG levels were significantly elevated $(17 \%)$ in the NAFLD 20 week group compared to the CTL group (CTL: $4.72 \pm 0.11 \mathrm{mmol} / \mathrm{L}$ vs. NAFLD 20 weeks: $5.53 \pm$ $0.15 \mathrm{mmol} / \mathrm{L}, p=0.001$ ) (Fig. 2d). HFD interruption was able to ameliorate FBG in 92\% (NAFLD 12 weeks: $5.10 \pm$ $0.12 \mathrm{mmol} / \mathrm{L}$ vs. NAFLD 20 weeks: $5.53 \pm 0.15 \mathrm{mmol} / \mathrm{L}$, $p=0.178$ ), while the diet intervention alongside the PM treatment led to additional effects, with FBG levels achieving similar values to the CTL group (NAFLD 12 weeks + PM: $4.65 \pm 0.20$ and CTL: $4.72 \pm 0.11 \mathrm{mmol} / \mathrm{L}, p>0.999)$ (Fig. 2d). 


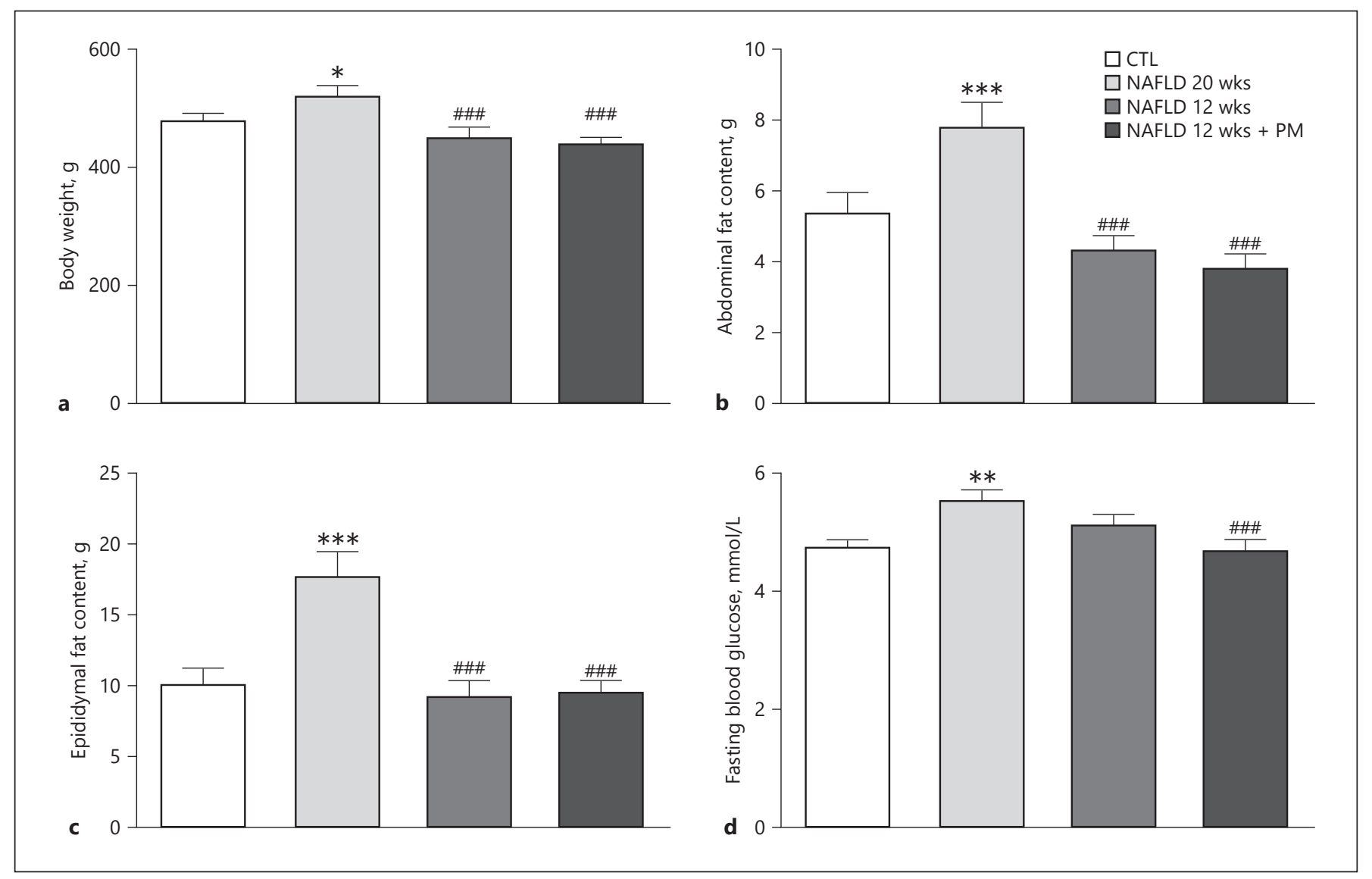

Fig. 2. Metabolic parameters of the rats used in this study. BW (a), abdominal and epididymal fat content (b, c, respectively), and FBG $(\mathbf{d})$. Values are expressed as means \pm SEM. ${ }^{*} p<0.05$ versus CTL, ${ }^{* *} p<0.01$ versus CTL, ${ }^{* * *} p<0.001$ versus CTL, \#\#\# $p<0.001$ versus NAFLD 20 weeks. NAFLD, nonalcoholic fatty liver disease; CTL, control; FBG, fasting blood glucose; BW, body weight.

Fig. 3. Representative image of the in vivo HMBF assessed by LSCI. Representative LSCI images and the setting of the ROI (circle) of the LLL of CTL (a), NAFLD 20 weeks (b), NAFLD 12 weeks (c) and NAFLD 12 weeks + PM (d) rats. The color scale represents the blood flow in APUs. Quantification of microcirculatory liver basal blood flow was measured by the LSCI method and expressed as APUs (e). ${ }^{* *} p<$ 0.01 versus CTL, \#\#p<0.01 versus NAFLD 20 weeks. LSCI, laser speckle contrast imaging; ROI, region of interest; LLL, left lateral liver lobe; APUs, arbitrary perfusion units; PM, pyridoxamine; NAFLD, nonalcoholic fatty liver disease; CTL, control; HMBF, hepatic microvascular blood flow.

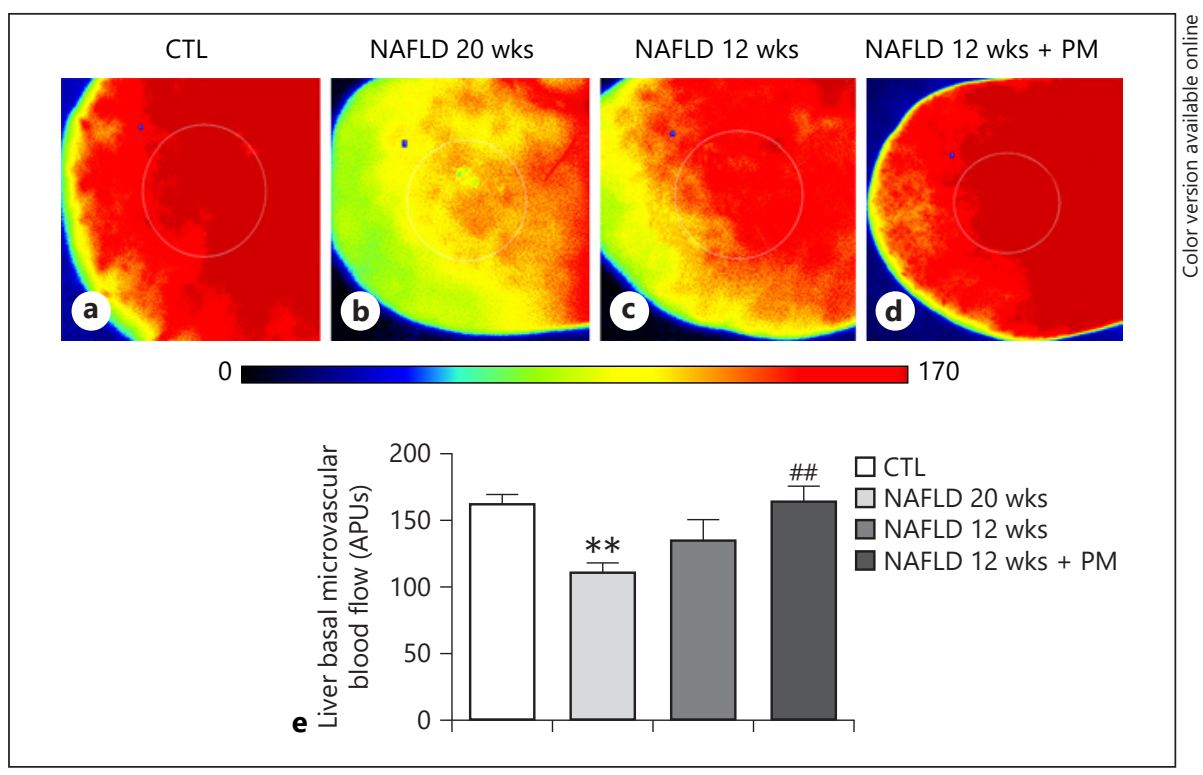




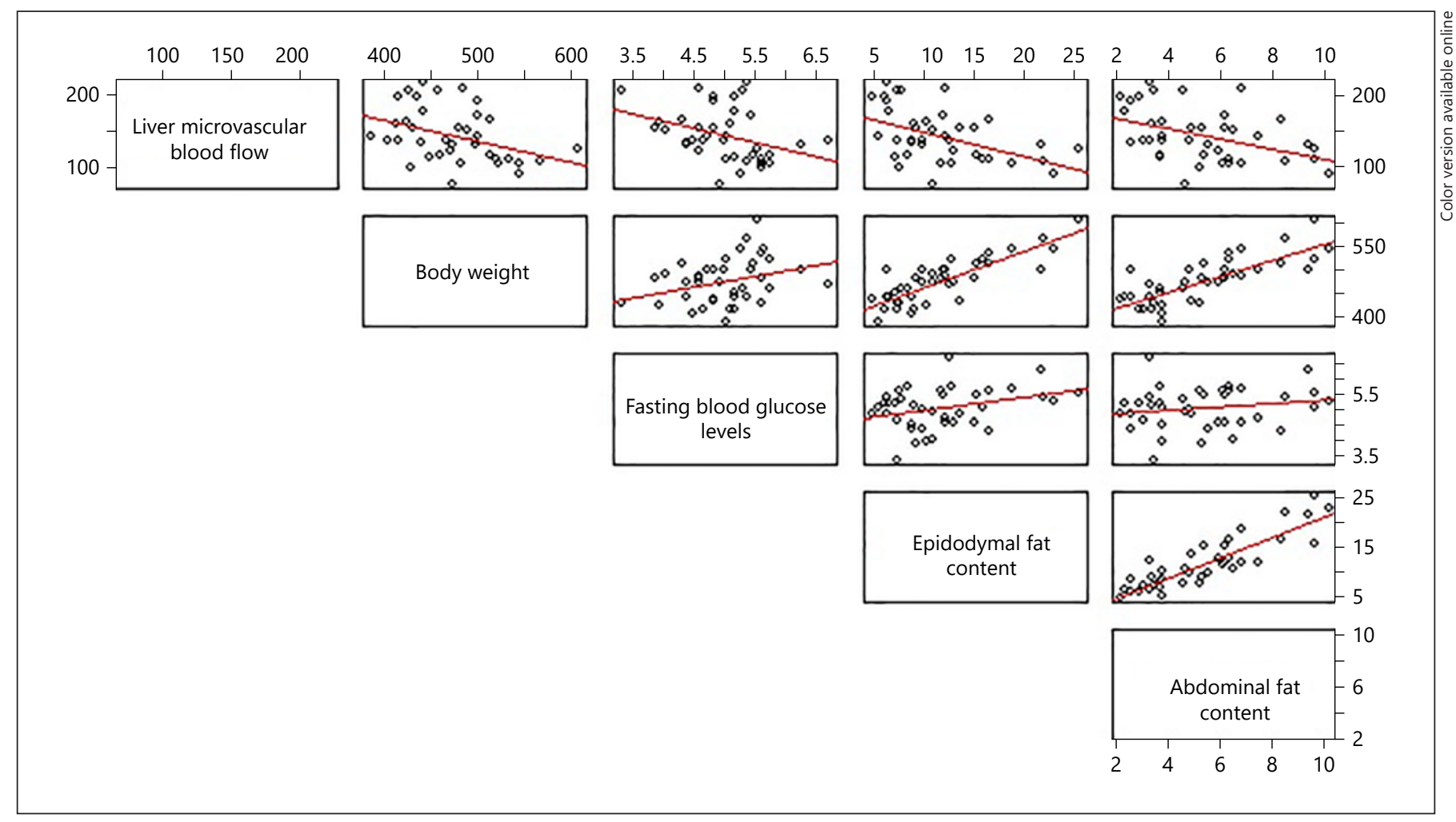

Fig. 4. Scatterplot matrix of the pairwise correlations of metabolic and microcirculatory parameters. Each matrix panel contains a scatterplot for a pair of parameters, and each dot in the scatterplot corresponds to a single measurement. BW, body weight; FBG, fasting blood glucose.

\section{Liver Microcirculatory Dysfunction}

Regarding the assessed microcirculatory parameters, the laser speckle contrast imaging analyses indicate a $31 \%$ decrease in liver microvascular blood flow in the NAFLD 20 week animal group when compared to CTL rats (CTL: $161.5 \pm 7.96$ APUs vs. NAFLD 20 weeks: $111.8 \pm 4.63$ APUs, $p=0.003$ ) (Fig. 3a-b, e). Although the diet composition changes resulted in an $85 \%$ improvement in the hepatic basal microvascular blood flow of the NAFLD 12 week group when compared to the NAFLD 20 week animals (NAFLD 12 weeks: $135.2 \pm 13.54$ APUs vs. NAFLD 20 weeks: $111.8 \pm 4.63$ APUs, $p=0.314$ ), the combined diet intervention and PM treatment led to a more pronounced beneficial effect of $100 \%$ blood flow recovery, achieving similar blood flow levels to control values (CTL: $161.5 \pm 7.96$ APUs vs. NAFLD 12 weeks + PM: $162.7 \pm$ 12.67 APUs, $p>0.999$ ) (Fig. 3c-e).

\section{Pearson Correlations}

Pearson's correlation analysis demonstrated that BW was positively correlated with epididymal fat content $(p<$
$0.001, r=0.83)$ and abdominal fat content $(p<0.001, r=$ $0.78)$. FBG levels were positively correlated with epididymal fat content $(p=0.041, r=0.33)$, while epididymal fat content was positively correlated with abdominal fat content $(p<0.001, r=0.88)$. Importantly, liver microvascular blood flow was negatively correlated with BW $(p=0.012$, $r=-0.39)$, FBG levels $(p=0.024, r=-0.35)$, and epididymal $(p=0.001, r=-0.48)$ and abdominal fat content $(p=$ $0.006, r=-0.43$ ) (Table 3; Fig. 4).

\section{Discussion/Conclusion}

The present study investigated whether metabolic and microcirculatory disorders associated with obesity and/ or NAFLD are modulated by caloric restriction alone or conjugated to PM treatment. Increased fat intake has been linked to obesity, insulin resistance, impaired postprandial lipid metabolism, and the development and progression of NAFLD [32]. Concerning obesity management, a weight management approach is also recom- 
mended for NAFLD patients [31, 43-47]. Lifestyle interventions, such as caloric restrictions, either low in carbohydrates or low in fats, is the first line of treatment for NAFLD. Sustained adherence to these lifestyle modifications however is difficult to achieve and maintain [48]. As the management of obesity and its hepatic complications through lifestyle modifications is an important challenge for most individuals, adjunct treatments are urgent and necessary [49].

PM, a vitamin B6 analog with antiglycative effects, has emerged as a promising nonpharmacological agent for the protection against the progressive tissue damage that occurs in diabetes and other diseases, such as schizophrenia, hyperlipidemia, chronic kidney disease, and myocardial infarction [50-53]. Vitamin B6 refers to 6 metabolically interchangeable compounds derived from pyridine, that is, pyridoxine, pyridoxal, pyridoxamine, and their respective phosphorylated forms. PM is found in food derived from animals and has already been described as acting, in its metabolically active form, as a cofactor for a variety of enzymes that participate in amino acid transamination, decarboxylation, and racemization [54-57]. In addition, $\mathrm{PM}$ acts by inhibiting the post-Amadori oxidative steps of the Maillard reaction through the binding of catalytic redox metal ions [58]. PM has proven effective against both systemic and hepatic changes in obesity-associated NAFLD [21]. A previous study by our group demonstrated that PM is able to reduce BW, liver leukocyte recruitment, and oxidative damage in animals presenting NAFLD [59].

In accordance with previous assessments by our group, the present study demonstrates that Wistar rat exposure to an HFD for 20 weeks induced an obesity-like phenotype, characterized by significant body adiposity associated with hyperglycemia $[20,21]$. In addition, we have previously shown that the same protocol concerning dietinduced obesity as applied herein also induces increases in systolic arterial blood pressure, hepatic triglycerides and cholesterol, and impairment of glucose and insulin metabolisms and steatoses, which make these animals suitable for NAFLD pathogenesis and intervention studies [20]. Herein, the diet intervention effects led to similar improvements in BW and epididymal and abdominal fat content, irrespective of PM supplementation. On the other hand, the association of PM with caloric restriction was more effective on glucose metabolism homeostasis recovery than the diet intervention alone. Fat content remains a more reliable adiposity measure than anthropometric measures, with VAT reported being associated with increased risks for cardiovascular and metabolic diseases

Vitamin Supplementation and Lifestyle Modifications in NAFLD Management
[15]. The literature supports the concept that VAT are compartments exhibiting a high metabolism rate and secreting free fatty acids, adipocytokines, and vasoactive substances that can negatively affect the liver through the hepatic portal vein circulation [60]. In addition, Sogabe et al. [61] reported that VAT increases may be one of the most important NAFLD predictors in women with metabolic syndrome. It has also been shown that lifestyle intervention consisting of caloric restriction with physical activity for 12 months in obese patients is able to decrease BW, visceral abdominal fat, liver fat content, blood pressure, and insulin resistance [62]. In a randomized controlled trial, a nutritional intervention aimed at reducing VAT in middle-aged adults led a substantial decrease in the percentage of hepatic fat content, resulting in moderate weight loss and beneficial cardiometabolic parameter effects [63]. In an assessed population of obese patients, a very low-calorie ketogenic diet exerted a positive effect in VAT decreases, ameliorating adiposity and blood biochemistry parameters [64]. The beneficial additional effects of PM can be explained by its function as an essential cofactor for enzymes involved in biological and metabolic activities, such as in the amino acid, fat, and glucose metabolisms [65, 66]. Maessen et al. [40] demonstrated that 18 weeks of PM treatment improved several obesity aspects, such as metabolic dysfunction, insulin resistance, and tissue inflammation in obese mice. Kim et al. [67] evaluated the antihyperglycemic potential of pyridoxal and its derivatives using Sprague-Dawley rats and reported that this nonpharmacological intervention decreased FBG levels by inhibiting carbohydrate hydrolysis and the activity of enzymes linked to glucose absorption, such as sucrase, maltase, and glucoamylase.

Liver microcirculation is essential for the physiological function of the entire organism, ensuring the supply of oxygen and nutrients to parenchymal tissue. In addition, it serves as a gateway for leukocytes in liver inflammation cases and is responsible for the clearance of toxic metabolites and foreign bodies from the bloodstream [68]. Dietary fat and obesity have negative impacts on microvascular health. HFD appears to promote endothelial dysfunction [66], and a high-saturated fat diet impairs endothelial vasodilation $[16,18,69,70]$.

Although the exact mechanisms remain unclear, in the liver, decreases in the size of the sinusoidal lumen is due, in part, to swollen hepatocyte "ballooning" as a consequence of the abnormal accumulation of triacylglycerol within the hepatocyte cytoplasm, which may cause partial or complete obstruction of the hepatic sinusoid space in up to $50 \%$ compared with the normal liver $[26,71]$. Lipid 
accumulation also occurs within the mitochondrial matrix, resulting in the production of superoxide and vasoactive metabolites that promote the activation of hepatic stellate cells [24]. Preclinical fatty liver studies have demonstrated that steatosis is inversely correlated with the total and microcirculatory hepatic blood flows [26], but the severity of steatosis has a more determinant impact on hepatic microcirculation than total liver blood flow [25]. Passarin et al. [72] demonstrated that Wistar Kyoto rats fed on a cafeteria diet exhibited overweight, arterial hypertension, hypertriglyceridemia, insulin resistance, and hepatic steatosis, all associated with hepatic endothelial dysfunction. Obese Zucker rats after schistosomiasis and reperfusion presented steatotic livers and abnormal microcirculation, manifested by a reduced sinusoidal density due to the decrease in the number of functional sinusoids and greater leukocyte adherence [27]. Recently, our group demonstrated that animals presenting obesity and NAFLD exhibit hepatic microcirculation disturbances, which can play an important role in NAFLD pathogenesis [20] and that a PM treatment for 8 weeks normalized the detected microcirculatory disturbances [21]. Of note, we report herein that caloric restriction and the PM treatment result in similar microcirculatory improvement effects, although within a significantly shorter period of time, that is, 2 weeks. In the present study, caloric restriction was able to partially improve liver microcirculation dysfunction, an important and original finding, while the diet modification associated with PM treatment led to a more pronounced effect on HMBF normalization than the diet intervention alone. Diet intervention as a strategy to reverse microcirculatory dysfunction had been assessed by several studies [73-77]. The additive benefit of PM supplementation on HMBF may be attributed to its ability to (a) improve metabolic parameters, (b) decrease hepatic AGE levels, (c) reduce ROS, (d) improve lipid peroxidation, and (e) decrease hepatic stellate cell activation [21]. To the best of our knowledge, this is the first time that the hepatoprotective effect of PM in association with a diet intervention has been shown in in vivo liver microcirculation.

NAFLD is a multifactorial disease comprising multiple physiological and biochemical events, including genetic, environmental, metabolic, and stress-related factors, even affecting extrahepatic organs and regulatory pathways [78]. In this context, hepatic microcirculatory abnormalities have emerged as an important risk factor for NAFLD progression and complications [20,72]. A clinical study evaluating the correlation between NAFLD and microvascular complications in type 2 diabetes mellitus reported that NAFLD was positively correlated with BMI, waist circumference, triglyceride levels, and FBG [79]. A preclinical study focusing on morphological features and microcirculation in NAFLD demonstrated that the degree of steatosis correlates with reduced blood flow speed in central veins, as well as in sinusoids [80]. We demonstrate herein that liver microcirculation blood flow is negatively correlated with BW, FBG, and VAT, suggesting the involvement of metabolic parameters in hepatic microcirculation impairment. The data reported in the present study substantiate the implementation of PM supplementation in association with lifestyle modifications as an effective intervention for the metabolic dysfunction and hepatic complications associated with obesity and NAFLD, controlling BW, visceral fat content and glycemia, and recovery to normal $\mathrm{HMBF}$.

\section{Statement of Ethics}

All experimental procedures were approved by the Oswaldo Cruz Foundation Animal Welfare Committee (CEUA license L-034/2016).

\section{Conflict of Interest Statement}

The authors have no conflicts of interest to declare.

\section{Funding Sources}

This study was supported by the Conselho Nacional de Desenvolvimento Científico e Tecnológico (CNPq 455.384/2014-2) and PAPES/Fiocruz (401.803/2015-5).

\section{Author Contributions}

Pereira E.N.G.D.S. and Daliry A. designed the research, performed the research, analyzed the data, and wrote the paper; Silvares R.R., Flores E.E.I., and Rodrigues K.L. performed the research and analyzed the data. All authors gave final approval and agreed to be accountable for their work. 


\section{References}

1 Nascimento AR, Machado M, de Jesus N, Gomes F, Lessa MA, Bonomo IT, et al. Structural and functional microvascular alterations in a rat model of metabolic syndrome induced by a high-fat diet. Obesity. 2013;21(10):204654.

2 World Health Organization. Obesity and overweight; 2020.

3 Endalifer ML, Diress G. Epidemiology, predisposing factors, biomarkers, and prevention mechanism of obesity: a systematic review. J Obes. 2020;2020:6134362.

4 Lee H, Lee IS, Choue R. Obesity, inflammation and diet. Pediatr Gastroenterol Hepatol Nutr. 2013;16(3):143-52.

5 Polyzos SA, Kountouras J, Mantzoros CS. Adipose tissue, obesity and non-alcoholic fatty liver disease. Minerva Endocrinol. 2017; 42(2):92-108

6 Pierantonelli I, Svegliati-Baroni G. Nonalcoholic fatty liver disease: basic pathogenetic mechanisms in the progression from NAFLD to NASH. Transplantation. 2019;103(1):e1-13.

7 Williams CD, Stengel J, Asike MI, Torres DM, Shaw J, Contreras M, et al. Prevalence of nonalcoholic fatty liver disease and nonalcoholic steatohepatitis among a largely middle-aged population utilizing ultrasound and liver biopsy: a prospective study. Gastroenterology. 2011;140(1):124-31.

8 Fan JG, Kim SU, Wong VW. New trends on obesity and NAFLD in Asia. J Hepatol. 2017; 67(4):862-73.

9 Silverman JF, O'Brien KF, Long S, Leggett N, Khazanie PG, Pories WJ, et al. Liver pathology in morbidly obese patients with and without diabetes. Am J Gastroenterol. 1990;85(10): 1349-55.

10 Bedogni G, Miglioli L, Masutti F, Tiribelli C, Marchesini G, Bellentani S. Prevalence of and risk factors for nonalcoholic fatty liver disease: the Dionysos nutrition and liver study. Hepatology. 2005;42(1):44-52.

11 Wree A, Kahraman A, Gerken G, Canbay A. Obesity affects the liver: the link between adipocytes and hepatocytes. Digestion. 2011; 83(1-2):124-33.

12 Thamer C, Machann J, Haap M, Stefan N, Heller E, Schnödt B, et al. Intrahepatic lipids are predicted by visceral adipose tissue mass in healthy subjects. Diabetes Care. 2004; 27(11):2726-9.

13 Marin P, Andersson B, Ottosson M, Olbe L, Chowdhury B, Kvist H, et al. The morphology and metabolism of intraabdominal adipose tissue in men. Metabolism. 1992;41(11):1242-8.

14 Parry SA, Hodson L. Influence of dietary macronutrients on liver fat accumulation and metabolism. J Investig Med. 2017;65(8): 1102-15.

15 Agbim U, Carr RM, Pickett-Blakely O, Dagogo-Jack S. Ethnic disparities in adiposity: focus on non-alcoholic fatty liver disease, visceral, and generalized obesity. Curr Obes Rep. 2019;8(3):243-54.
16 Buie JJ, Watson LS, Smith CJ, Sims-Robinson C. Obesity-related cognitive impairment: the role of endothelial dysfunction. Neurobiol Dis. 2019;132:104580.

17 Alramadan MJ, Magliano DJ, Alhamrani HA, Alramadan AJ, Alameer SM, Amin GM, et al. Lifestyle factors and macro- and micro-vascular complications among people with type 2 diabetes in Saudi Arabia. Diabetes Metab Syndr. 2019 Jan-Feb;13(1):484-91.

18 Rutkowski JM, Davis KE, Scherer PE. Mechanisms of obesity and related pathologies: the macro- and microcirculation of adipose tissue. FEBS J. 2009;276(20):5738-46.

19 Kobayasi R, Akamine EH, Davel AP, Rodrigues MA, Carvalho CR, Rossoni LV. Oxidative stress and inflammatory mediators contribute to endothelial dysfunction in highfat diet-induced obesity in mice. J Hypertens. 2010;28(10):2111-9.

20 Pereira ENGDS, Silvares RR, Flores EEI, Rodrigues KL, Ramos IP, da Silva IJ, et al. Hepatic microvascular dysfunction and increased advanced glycation end products are components of non-alcoholic fatty liver disease. PLoS One. 2017;12(6):e0179654.

21 Pereira ENGDS, Silvares RR, Flores EEI, Rodrigues KL, Daliry A. Pyridoxamine improves metabolic and microcirculatory complications associated with nonalcoholic fatty liver disease. Microcirculation. 2020;27(3):e12603.

22 Suboc TM, Dharmashankar K, Wang J, Ying R, Couillard A, Tanner MJ, et al. Moderate obesity and endothelial dysfunction in humans: influence of gender and systemic inflammation. Physiol Rep. 2013;1(3):e00058.

23 Csige I, Ujvárosy D, Szabó Z, Lőrincz I, Paragh G, Harangi M, et al. The impact of obesity on the cardiovascular system. J Diabetes Res. 2018;2018:3407306.

24 Brock RW, Dorman RB. Obesity, insulin resistance and hepatic perfusion. Microcirculation. 2007;14(4-5):339-47.

25 Ijaz S, Yang W, Winslet MC, Seifalian AM. Impairment of hepatic microcirculation in fatty liver. Microcirculation. 2003;10(6):44756.

26 Seifalian AM, Piasecki C, Agarwal A, Davidson BR. The effect of graded steatosis on flow in the hepatic parenchymal microcirculation. Transplantation. 1999;68(6):780-4.

27 Sun CK, Zhang XY, Zimmermann A, Davis G, Wheatley AM. Effect of ischemia-reperfusion injury on the microcirculation of the steatotic liver of the Zucker rat. Transplantation. 2001;72(10):1625-31.

28 Barrera F, George J. The role of diet and nutritional intervention for the management of patients with NAFLD. Clin Liver Dis. 2014; 18(1):91-112.

29 Kaliora AC, Gioxari A, Kalafati IP, Diolintzi A, Kokkinos A, Dedoussis GV. The effectiveness of mediterranean diet in nonalcoholic fatty liver disease clinical course: an intervention study. J Med Food. 2019;22(7):729-40.
30 Plaz Torres MC, Aghemo A, Lleo A, Bodini G, Furnari M, Marabotto E, et al. Mediterranean diet and NAFLD: what we know and questions that still need to be answered. Nutrients. 2019;11(12):2971.

31 Romero-Gomez M, Zelber-Sagi S, Trenell M. Treatment of NAFLD with diet, physical activity and exercise. J Hepatol. 2017;67(4):82946.

32 Fan JG, Cao HX. Role of diet and nutritional management in non-alcoholic fatty liver disease. J Gastroenterol Hepatol. 2013;28(Suppl 4):81-7.

33 Chalasani N, Younossi Z, Lavine JE, Charlton M, Cusi K, Rinella M, et al. The diagnosis and management of nonalcoholic fatty liver disease: practice guidance from the American Association for the study of liver diseases. Hepatology. 2018;67(1):328-57.

34 Promrat K, Kleiner DE, Niemeier HM, Jackvony E, Kearns M, Wands JR, et al. Randomized controlled trial testing the effects of weight loss on nonalcoholic steatohepatitis. Hepatology. 2010;51(1):121-9.

35 European Association for the Study of the Liver, European Association for the Study of Diabetes and European Association for the Study of Obesity. EASL-EASD-EASO clinical practice guidelines for the management of non-alcoholic fatty liver disease. J Hepatol. 2016;64(6):1388-402.

36 Chitturi S, Wong VW, Chan WK, Wong GL, Wong SK, Sollano J, et al. The Asia-Pacific working party on non-alcoholic fatty liver disease guidelines 2017-part 2: management and special groups. J Gastroenterol Hepatol. 2018;33(1):86-98.

37 Ho LT, Chiang HL, Tsai H, Chou TY, Kwok CF. A therapeutic trial of bezafibrate on patients with hyperlipidemia with or without diabetes mellitus. Proc Natl Sci Counc Repub China B. 1984;8(3):240-5.

38 Hagiwara S, Gohda T, Tanimoto M, Ito T, Murakoshi M, Ohara I, et al. Effects of pyridoxamine (K-163) on glucose intolerance and obesity in high-fat diet C57BL/6J mice. Metab Clin Exp. 2009;58(7):934-45.

39 Oh S, Ahn H, Park H, Lee JI, Park KY, Hwang $\mathrm{D}$, et al. The attenuating effects of pyridoxamine on adipocyte hypertrophy and inflammation differ by adipocyte location. J Nutr Biochem. 2019;72:108173.

40 Maessen DE, Brouwers O, Gaens KH, Wouters K, Cleutjens JP, Janssen BJ, et al. Delayed intervention with pyridoxamine improves metabolic function and prevents adipose tissue inflammation and insulin resistance in high-fat diet-induced obese mice. Diabetes. 2016;65(4):956-66

41 Li CH, Ge XL, Pan K, Wang PF, Su YN, Zhang $\mathrm{AQ}$. Laser speckle contrast imaging and oxygen to see for assessing microcirculatory liver blood flow changes following different volumes of hepatectomy. Microvasc Res. 2017; 110:14-23. 
42 Wu Y, Ren J, Zhou B, Ding C, Chen J, Wang $\mathrm{G}$, et al. Laser speckle contrast imaging for measurement of hepatic microcirculation during the sepsis: a novel tool for early detection of microcirculation dysfunction. Microvasc Res. 2015;97:137-46.

43 Jensen MD, Ryan DH, Apovian CM, Ard JD, Comuzzie AG, Donato KA, et al. 2013 AHA/ ACC/TOS guideline for the management of overweight and obesity in adults: a report of the American College of Cardiology/American Heart Association Task Force on Practice Guidelines and the Obesity Society. Circulation. 2014;129(25 Suppl 2):S102-38.

44 Yumuk V, Tsigos C, Fried M, Schindler K, Busetto L, Micic D, et al. European guidelines for obesity management in adults. Obes Facts. 2015;8(6):402-24.

45 Ryan DH, Kahan S. Guideline recommendations for obesity management. Med Clin North Am. 2018;102(1):49-63.

46 Smethers AD, Rolls BJ. Dietary management of obesity: cornerstones of healthy eating patterns. Med Clin North Am. 2018;102(1):10724.

47 Vilar-Gomez E, Martinez-Perez Y, Calzadilla-Bertot L, Torres-Gonzalez A, Gra-Oramas B, Gonzalez-Fabian L, et al. Weight loss through lifestyle modification significantly reduces features of nonalcoholic steatohepatitis. Gastroenterology. 2015;149(2):367-78. e5; .

48 Elfhag K, Rössner S. Who succeeds in maintaining weight loss? A conceptual review of factors associated with weight loss maintenance and weight regain. Obes Rev. 2005; 6(1):67-85.

49 Hensley RD. Primary care management of obesity: individualized treatment strategies. Nurse Pract. 2018;43(7):41-8.

50 Voziyan PA, Hudson BG. Pyridoxamine as a multifunctional pharmaceutical: targeting pathogenic glycation and oxidative damage. Cell Mol Life Sci. 2005;62(15):1671-81.

51 Chetyrkin SV, Kim D, Belmont JM, Scheinman JI, Hudson BG, Voziyan PA. Pyridoxamine lowers kidney crystals in experimental hyperoxaluria: a potential therapy for primary hyperoxaluria. Kidney Int. 2005;67(1):5360.

52 Itokawa $M$, Miyashita $M$, Arai $M$, Dan T, Takahashi K, Tokunaga T, et al. Pyridoxamine: a novel treatment for schizophrenia with enhanced carbonyl stress. Psychiatry Clin Neurosci. 2018;72(1):35-44.

53 Deluyker D, Ferferieva V, Driesen RB, Verboven M, Lambrichts I, Bito V. Pyridoxamine improves survival and limits cardiac dysfunction after MI. Sci Rep. 2017;7(1):16010.

54 Percudani R, Peracchi A. The B6 database: a tool for the description and classification of vitamin B6-dependent enzymatic activities and of the corresponding protein families. BMC Bioinformatics. 2009; 10:273.

55 Raboni S, Spyrakis F, Campanini B, Amadasi A, Bettati S, Peracchi A, et al. 7.10: pyridoxal
5 -phosphate-dependent enzymes: catalysis, conformation, and genomics. In: Comprehensive Natural Products II. 2010. Vol. 7; p. 273-350.

56 Adrover M, Vilanova B, Frau J, Muñoz F, Donoso J. The pyridoxamine action on amadori compounds: a reexamination of its scavenging capacity and chelating effect. Bioorg Med Chem. 2008;16(10):5557-69.

57 Nomi Y, Otsuka Y. Isolation, identification, and proposed formation mechanism of a novel hydrophilic compound formed by Maillard reaction between pyridoxamine and pentose. Sci Rep. 2020;10(1):1823.

58 Skrypnyk NI, Voziyan P, Yang H, de Caestecker CR, Theberge MC, Drouin M, et al. Pyridoxamine reduces postinjury fibrosis and improves functional recovery after acute kidney injury. Am J Physiol Renal Physiol. 2016; 311(2):F268-77.

59 Rangel Silvares R, Nunes Goulart da Silva Pereira E, Eduardo Ilaquita Flores E, Lino Rodrigues K, Ribeiro Silva A, Gonçalves-de-Albuquerque $\mathrm{CF}$, et al. High-fat diet-induced kidney alterations in rats with metabolic syndrome: endothelial dysfunction and decreased antioxidant defense. Diabetes Metab Syndr Obes. 2019;12:1773-81.

60 Fox CS, Massaro JM, Hoffmann U, Pou KM, Maurovich-Horvat P, Liu CY, et al. Abdominal visceral and subcutaneous adipose tissue compartments: association with metabolic risk factors in the Framingham Heart Study. Circulation. 2007;116(1):39-48.

61 Sogabe M, Okahisa T, Tsujigami K, Fukuno $\mathrm{H}$, Hibino S, Yamanoi A. Visceral fat predominance is associated with non-alcoholic fatty liver disease in Japanese women with metabolic syndrome. Hepatol Res. 2014;44(5): $515-22$.

62 Goodpaster BH, Delany JP, Otto AD, Kuller L, Vockley J, South-Paul JE, et al. Effects of diet and physical activity interventions on weight loss and cardiometabolic risk factors in severely obese adults: a randomized trial. JAMA. 2010;304(16):1795-802.

63 Gepner Y, Shelef I, Komy O, Cohen N, Schwarzfuchs D, Bril N, et al. The beneficial effects of Mediterranean diet over low-fat diet may be mediated by decreasing hepatic fat content. J Hepatol. 2019;71(2):379-88.

64 Valenzano A, Polito R, Trimigno V, Di Palma A, Moscatelli F, Corso G, et al. Effects of very low calorie ketogenic diet on the orexinergic system, visceral adipose tissue, and ROS production. Antioxidants. 2019;8(12):643.

65 Liu Z, Li P, Zhao ZH, Zhang Y, Ma ZM, Wang SX. Vitamin B6 prevents endothelial dysfunction, insulin resistance, and hepatic lipid accumulation in apoe $(-/-)$ mice fed with high-fat diet. J Diabetes Res. 2016;2016: 1748065.

66 Metz TO, Alderson NL, Thorpe SR, Baynes JW. Pyridoxamine, an inhibitor of advanced glycation and lipoxidation reactions: a novel therapy for treatment of diabetic complica- tions. Arch Biochem Biophys. 2003;419(1): 41-9.

67 Kim HH, Kang YR, Lee JY, Chang HB, Lee $\mathrm{KW}$, Apostolidis E, et al. The postprandial anti-hyperglycemic effect of pyridoxine and its derivatives using in vitro and in vivo animal models. Nutrients. 2018;10(3):285.

68 Vollmar B, Menger MD. The hepatic microcirculation: mechanistic contributions and therapeutic targets in liver injury and repair. Physiol Rev. 2009;89(4):1269-339.

69 Keogh JB, Grieger JA, Noakes M, Clifton PM. Flow-mediated dilatation is impaired by a high-saturated fat diet but not by a high-carbohydrate diet. Arterioscler Thromb Vasc Biol. 2005;25(6):1274-9.

70 Virdis A, Masi S, Colucci R, Chiriacò M, Uliana M, Puxeddu I, et al. Microvascular endothelial dysfunction in patients with obesity. Curr Hypertens Rep. 2019;21(4):32.

71 Sato N, Eguchi H, Inoue A, Matsumura T, Kawano S, Kamada T. Hepatic microcirculation in Zucker fatty rats. Adv Exp Med Biol. 1986;200:477-83.

72 Pasarin M, La Mura V, Gracia-Sancho J, García-Calderó H, Rodríguez-Vilarrupla M, García-Pagán JC, et al. Sinusoidal endothelial dysfunction precedes inflammation and fibrosis in a model of NAFLD. PLoS One. 2012; 7(4): 32785.

73 Woo KS, Chook P, Yu CW, Sung RY, Qiao M, Leung SS, et al. Effects of diet and exercise on obesity-related vascular dysfunction in children. Circulation. 2004;109(16):1981-6.

74 Rogerson D, McNeill S, Könönen H, Klonizakis M. Encouraging effects of a short-term, adapted Nordic diet intervention on skin microvascular function and skin oxygen tension in younger and older adults. Nutrition. 2018; 49:96-101.

75 Csipo T, Fulop GA, Lipecz A, Tarantini S, Kiss $\mathrm{T}$, Balasubramanian $\mathrm{P}$, et al. Short-term weight loss reverses obesity-induced microvascular endothelial dysfunction. Geroscience. 2018 Jun 18;40(3):337-46.

76 Bigornia SJ, Farb MG, Tiwari S, Karki S, Hamburg NM, Vita JA, et al. Insulin status and vascular responses to weight loss in obesity. J Am Coll Cardiol. 2013;62(24):2297-305.

77 Montero D, Walther G, Perez-Martin A, Mercier CS, Gayrard S, Vicente-Salar N, et al. Effects of a lifestyle program on vascular reactivity in macro- and microcirculation in severely obese adolescents. J Clin Endocrinol Metab. 2014;99(3):1019-26.

78 Byrne CD, Targher G. NAFLD: a multisystem disease. J Hepatol. 2015;62(1 Suppl):S47-64.

79 Lv WS, Sun RX, Gao YY, Wen JP, Pan RF, Li $\mathrm{L}$, et al. Nonalcoholic fatty liver disease and microvascular complications in type 2 diabetes. World J Gastroenterol. 2013;19(20): 3134-42.

80 Rosenstengel S, Stoeppeler S, Bahde R, Spiegel HU, Palmes D. Type of steatosis influences microcirculation and fibrogenesis in different rat strains. J Invest Surg. 2011;24(6):273-82. 